\title{
CONSEJERÍA EN VIDA SANA Y CAMBIO DE CONDUCTAS EN ESCOLARES OBESOS: INTERVENCIÓN CONTROLADA EN MADRES/CUIDADORAS
}

\author{
COUNSELING ON HEALTHY LIFESTYLES AND \\ BEHAVIORAL CHANGE IN OBESE SCHOOL CHILDREN: \\ CONTROLLED INTERVENTION IN MOTHERS/CARETAKERS
}

\author{
Álvaro Castillo C., Juliana Kain B. \\ Instituto de Nutrición y Tecnología de los Alimentos (INTA) \\ Universidad de Chile. Santiago, Chile.
}

\begin{abstract}
Obesity is the main nutritional problem among Chilean children. Behavioral changes through the stage of change model have been proposed as a low cost prevention alternative that has greater impact than the informative model. The purpose of this study was to evaluate the effect of counseling on healthy lifestyles on the behavioral changes of obese children 6 to 9 years of age. A quasi-experimental pilot study including mothers/caretakers $(N=50)$ of obese children from public schools was carried out. The intervention consisted in three home-based counseling sessions to the mothers/caretakers during a 16-week period. The main results indicated that counseling contributed to decreased daily television time and improved the nutritional quality of the foods brought from home by children from the intervention group compared to children from the control group. Counseling is an effective prevention strategy whose effects need to be confirmed in a randomized study including a larger sample.

Key words: Counseling; childhood obesity; nutritional conduct; physical activity; mothers/carers.
\end{abstract}

Este trabajo fue recibido el 10 de Noviembre de 2009 y aceptado para ser publicado el 14 de Mayo de 2010.

\section{INTRODUCCIÓN}

La alta prevalencia de obesidad en preescolares y escolares en Chile constituye un problema de salud pública que ha preocupado a las autoridades por su continuo aumento y por su asociación con enfermedades crónicas no transmisibles (ECNT) en la vida adulta (1). El año 2008, la prevalencia de obesidad en escolares de primer año básico de escuelas públicas alcanzó el 20,4 $\%$, según cifras aportados por JUNAEB (2), lo cual ha significado un replanteamiento de las metas nutricionales que se pretendía alcanzar el 2010 (de un 16\% a un 12\%) en esta población de niños (3).

La prevención de enfermedades crónicas y el control de los factores de riesgo asociados a éstas, son parte del enfoque asumido por el sector salud para disminuir la creciente morbimortalidad por enfermedad cardiovascular, hipertensión arterial y diabetes tipo 2 (4). El control del peso corporal desde edades tempranas se reconoce como un eje de intervención en promoción de salud que pueden conducir a una mejora de las actuales cifras de obesidad $(5,6)$.

Una de las alternativas de intervención que se han propuesto, y que toma distancia del enfoque informativo característico del modelo biomédico, es el modelo motivacional/ progresivo de las etapas de cambio (muchas veces denominado consejería), el cual se reconoce como una alternativa válida, de bajo costo y de mayor impacto (7).

\section{Consejería en salud}

La consejería es una estrategia de asesoría que está basada en modelos teórico/ conceptuales desarrollados hace varias décadas atrás. Uno de estos modelos es el transteórico desarrollado por Prochaska y Di clemente, cuya principal diferencia respecto a otras formas de asesoría es la de entender el cambio de conducta como 
algo progresivo, y cuyas etapas de cambio se pueden identificar y trabajar individualmente. Los autores identificaron las etapas de acuerdo al interés o compromiso con el cambio, e identificaron cinco fases dentro de éste: precontemplación, contemplación, preparación para la acción, acción y mantención, y donde cada una de estas etapas tiene características propias, con intervenciones específicas para cada una de ellas (8). En esta investigación se propuso emplear el Manual de Consejería en Vida Sana desarrollado por Albala y cols. (9) que además incorpora otros aspectos conceptuales de la conducta como el desarrollado por Green (10), quien identificó que en el proceso de cambio existen factores que él denominó como predisponentes, facilitadores y reforzantes.

En este estudio, los hábitos alimentarios y la actividad física son las dos dimensiones intervenidas con el objetivo de producir cambios de conducta que favorezcan estilos de vida más saludables. El objetivo de este estudio fue evaluar el efecto de implementar el modelo de Consejería en Vida Sana en madres/cuidadoras de escolares obesos, en producir cambios de conducta relacionados con alimentación y actividad física.

\section{SUJETOS Y MÉTODOS}

Este es un estudio piloto, con diseño cuasi-experimental y auxiliar del proyecto Macul de Prevención de Obesidad en Escuelas Municipalizadas (11), desarrollado por el Instituto de Nutrición y Tecnología de los Alimentos de la Universidad de Chile. Ambos estudios cuentan con la aprobación de la Corporación Municipal de Educación.

\section{Participantes}

La muestra fue por conveniencia e incluyó la total de madres/cuidadoras de niños obesos que accedieron ingresar al estudio y que cumplieran con los criterios de inclusión (50 de 99). Se realizó una medición de peso y talla durante los meses de Abril y Mayo del 2008, y su clasificación nutricional se realizó de acuerdo a los estándares de la OMS del 2007 (12). Se contactó telefónicamente y vía comunicación del colegio a las madres y/o apoderados de los niños obesos (IMC $>2 \mathrm{DE}$ ) de seis de las siete escuelas que forman parte del Proyecto Macul.

Los criterios de inclusión fueron: cursar entre $1^{\circ}$ a $3^{\circ}$ básico (edad entre 6 y 9 años); ser obeso (IMC $>2 \mathrm{DE}$ ); no vivir en un hogar infantil y no haber sido parte de un programa o tratamiento nutricional en el último año.

Previo al ingreso en el estudio, los participantes leyeron y firmaron un documento de consentimiento informado.

\section{Variables estudiadas}

Variables resultado: consumo y calidad de las colaciones que los niños llevan frecuentemente a la escuela; cantidad de dinero con el que disponen los días de clases; tiempo que los niños destinan a ver televisión los días de semana e inscripción a talleres polideportivos o actividad física extraescolar.

Variables intermedias: etapas de cambio de conducta de las cuatro variables dependientes, entendidas como subrogante de la intención de cambio.

Variables descriptivas: Edad de la madre/cuidadora; nivel socioeconómico (NSE).

Las variables como peso, talla, sexo, IMC y edad fueron evaluadas como parte del Proyecto Macul.

\section{Instrumentos}

El cuestionario se aplicó a las madres/cuidadoras de los niños por entrevistadoras previamente entrenadas. El tiempo aproximado para la aplicación de este cuestionario fue entre 15 a 20 minutos. Para evaluar el consumo y calidad de las colaciones se aplicó un cuestionario modificado de Olivares et al. (13), mientras que el tiempo destinado a ver televisión se determinó preguntando a los apoderados por cada uno de los programas que el niño ve habitualmente los días de semana y el tiempo aproximado que los ve. En cuanto a si el niño estaba inscrito en un taller deportivo dentro de la escuela, se contrastó la información entregada por los apoderados con los registros de inscripción y asistencia a los talleres, disponibles en la Corporación de Educación de la Municipalidad de Macul.

La evaluación de las etapas de cambio de conducta se realizó a través de un cuestionario modificado para evaluar intención de cambio de conducta (etapas de cambio según modelo transteórico), elaborado por el Instituto Nacional del Cáncer y el Instituto Nacional de Salud de Estado Unidos La caracterización socioeconómica de los participantes se obtuvo a través del cuestionario de evaluación socioeconómica ESOMAR, (15) que contempla actividad ocupacional y educación del jefe de hogar, y en caso de encontrarse económicamente inactivo (desempleado, jubilado) se utilizó una batería de bienes. Las demás variables fueron evaluadas con un cuestionario ad-hoc diseñado para estos propósitos.

\section{Intervención}

Al grupo intervenido se le realizaron 3 consejerías individuales en el hogar a la madre/cuidadora cada 5 semanas, siguiendo el protocolo de intervención descrito en el manual de Consejería en Vida Sana y realizadas por autor principal del estudio, previamente capacitado y entrenado. El período de intervención duró 16 semanas 
a partir de junio del año 2008 hasta octubre del mismo año. El seguimiento de los participantes se realizó vía telefónica. La duración de las consejería fue de alrededor de 20 a 25 minutos, dentro de los cuales se abordó el cambio de conducta desde un enfoque progresivo y diferenciado de acuerdo a la intención de cambio evaluada al inicio y a las características individuales de cada persona. Los puntos que se tomaron en cuenta al momento de estructurar una intervención fueron la etapa de cambio en la que se encontraban los participantes y los factores predisponentes, facilitadores y reforzantes de la conducta (10), sondeados al inicio de la intervención.

El fundamento de las recomendaciones nutricionales se basó en la Guía Alimentaria desarrollada por el Instituto de Nutrición y Tecnología de los Alimentos de la Universidad de Chile (INTA) para escolares entre 6 y 10 años de edad (16). El grupo control recibió la guía ya mencionada, más una guía informativa para aumentar el consumo de frutas y verduras desarrollada por el INTA en el marco del programa "5 al día" (17).

\section{Análisis}

Se realizó una estadística descriptiva para mostrar la distribución de las variables evaluadas.

Para el análisis de las variables categóricas y dicotómicas se utilizó el Test Exacto de Fisher. Para comparar el porcentaje de cambio se dicotomizaron las variables de acuerdo a los criterio definidos como "aceptables" según los objetivos específicos del estudios. Se consideró como aceptable que los niños llevaran 3 o más colaciones desde el hogar, que llevaran hasta $\$ 100$ (aproximadamente 20 centavos de dólar) a la escuela y que el tiempo destinado a ver televisión no superara los 120 minutos diarios.

El cambio en las conductas estudiadas se analizaron categorizando el diferencial de cambio en tres categorías: (0) no se modificó la conducta; (1) se modificó en sentido negativo, es decir, hubo un cambio en el sentido contrario al buscado y; (2) se modificó la conducta en sentido positivo, es decir, se logró el cambio buscado. El mismo análisis se realizó con las variables intermedias.

Se utilizaron los paquetes estadísticos SPSS versión 11,5 y Stata versión 10,0. El nivel de significación utilizado fue el 0,05 .

\section{RESULTADOS}

La prevalencia de obesidad en los niños de $1^{\circ}$ a $3^{\circ}$ básico de las seis escuelas fue de un 15,9\% en la evaluación realizada en abril del 2008 y varió entre un 12\% y un $19,4 \%$, sin diferencia significativas entre los colegios $(\mathrm{p}=0,99)$. El puntaje $\mathrm{z}$ de IMC no varió entre los grupos (media 2,65 y desviación estándar (DE) 0,58 v/s 2,77 y DE $0,78, p=0,382)$ ni entre las escuelas $(\mathrm{p}=0,152)$.
La muestra que participó en este estudio fue de 50 madres/cuidadoras de niños obesos (25 en cada grupo), una vez excluidos los casos que no cumplían con los criterios de inclusión. De estos 50 sujetos ingresados y visitados al inicio, uno no logró ser contactado al final del estudio.

De los apoderados entrevistados, $98 \%$ correspondía a mujeres y dos de ellas eran parientes cercanos (tía y bisabuela) con la custodia de los niños. En la tabla 1 se muestran las características de ambos grupos al inicio del estudio. Si bien se observó una diferencia en el promedio de edad y los minutos de televisión entre los grupos, al estratificar por curso no hubo diferencias significativas. En la tabla 2 se muestra la etapa de cambio en la que se encontraban las madres del grupo control y del grupo intervenido al inicio del estudio.

En la tabla 3 se muestra la dirección del cambio y el porcentaje de niños que realizaron dicho cambio. Las variables que mostraron mayor cambio fueron el tipo de colación y los minutos de televisión, cambio que fue más acentuado en el grupo intervenido. La inscripción a talleres polideportivos tuvo un leve cambio positivo en el grupo control.

En relación a las variables intermedias, es decir, a la intención de cambio de conducta, se realizó el mismo análisis que en las variables anteriores. En la tabla 4 se observa que el porcentaje de variación en la intención de cambio de conducta. No hubo diferencias en ninguno de los grupos, aunque la tendencia al cambio fue mayor en los intervenidos, con excepción de la inscripción a los talleres deportivos.

\section{DISCUSIÓN}

En este estudio comparamos una intervención acotada, consistente en la entrega de material escrito v/s una intervención basada en entrevistas y orientadas a trabajar aquellos aspectos relacionados con la motivación en el cambio de conducta. Si bien los cambios observados fueron leves, se observó una tendencia al cambio favorable en ambos grupos, especialmente en los intervenidos. Esto sugiere que otras intervenciones con este modelo conceptual pudieran alcanzar resultados favorables en algunas conductas.

Se sabe que en Chile la dieta de los niños incluye snacks dulces y salados con un alto contenido calórico $(13,18)$, por lo que proponer una estrategia para mejorar el contenido de esas colaciones resulta prioritario, especialmente con los antecedentes vistos en otros países, donde las intervenciones realizadas a padres han tenido resultados positivos (19). Según un estudio realizado en Estados Unidos (20) donde se analizó la evolución del consumo de snacks en los niños, se vio el año 1996 que 
$83 \%$ y $96 \%$ (según el origen étnico) de los niños entre 6 y 11 años consumían en promedio 2 snacks diarios, lo cual equivale a 462 kilocalorias o al $24 \%$ de la energía requerida diariamente siendo similar en Chile la situación.

Cuando se planteó el objetivo de aumentar la proporción de niños que realiza actividad física, el principal obstáculo tuvo que ver con los espacios y tiempos para hacerlo, además de la supervisión que éstos requerían.
Los talleres polideportivos ofrecidos por la Corporación de Educación de la Municipalidad se presentaron como una gran oportunidad para fomentar la actividad física en un ambiente protegido, con la supervisión de un profesor de educación física contratado especialmente para esos propósitos y de forma gratuita. Pero como se observó en los resultados, la variable que tuvo menos cambios fue la inscripción a los talleres polideportivos, aumentando

\section{TABLA 1}

Características basales de los grupos intervenido y control

\begin{tabular}{|c|c|c|c|}
\hline & $\begin{array}{c}\text { Control } \\
(\mathrm{N}=25)\end{array}$ & $\begin{array}{l}\text { Intervenido } \\
\qquad(\mathbf{N}=\mathbf{2 5})\end{array}$ & $\mathbf{p}$ \\
\hline \multicolumn{4}{|l|}{$\operatorname{Sexo} \mathrm{n}(\%)$} \\
\hline Masculino & $14(56,0)$ & $15(60,0)$ & $1,00 \ddagger$ \\
\hline Femenino & $11(44,0)$ & $10(40,0)$ & \\
\hline Edad niños x(DE) & $7,62(0,89)$ & $8,08(0,99)$ & $0,09 \dagger$ \\
\hline zIMC niños. x(DE) & $2,65(0,53)$ & $2,82(0,98)$ & $0,65 \dagger$ \\
\hline Edad apoderado $x(D E)$ & $36,10(7,32)$ & $36,81(8,31)$ & $0,76 \dagger$ \\
\hline \multicolumn{4}{|l|}{$\operatorname{NSE} n(\%)$} \\
\hline Baja & $5(20,0)$ & $6(24,0)$ & $0,582 \div$ \\
\hline Media-baja & $12(48,0)$ & $14(56,0)$ & \\
\hline Media & $7(28,0)$ & $3(12,0)$ & \\
\hline Media-alta & $1(4,0)$ & $2(8,0)$ & \\
\hline \multicolumn{4}{|l|}{ Lleva colación n(\%) } \\
\hline Nunca & $4(16,0)$ & $6(24,0)$ & $0,742 \ddagger$ \\
\hline 1 a 2 veces por semana & $1(4,0)$ & $0(0,0)$ & \\
\hline 3 a 4 veces por semana & $2(8,0)$ & $3(12,0)$ & \\
\hline Siempre & $18(72,0)$ & $16(64,0)$ & \\
\hline \multicolumn{4}{|l|}{ Tipo de colación n $(\%)$} \\
\hline No Saludable & $11(52,6)$ & $12(63,2)$ & $0.569 \ddagger$ \\
\hline Saludable & $10(47,4)$ & $7(37,4)$ & \\
\hline \multicolumn{4}{|l|}{ Lleva dinero $\mathrm{n}(\%)$} \\
\hline Nunca & $13(52,0)$ & $5(20,0)$ & $0,107 \ddagger$ \\
\hline 1 a 2 veces por semana & $4(16,0)$ & $9(36,0)$ & \\
\hline 3 a 4 veces por semana & $3(12,0)$ & $3(12,0)$ & \\
\hline Siempre & $5(20,0)$ & $8(32,0)$ & \\
\hline \multicolumn{4}{|c|}{ Inscripción taller polideportivo $\mathrm{n}(\%)$} \\
\hline $\mathrm{Si}$ & $4(16,0)$ & $5(20,0)$ & $1,00 \ddagger$ \\
\hline No & $21(84,0)$ & $20(80,0)$ & \\
\hline Minutos TV. x(DE) & $139,3(74,63)$ & $190,8(83,74)$ & $0,03 \dagger$ \\
\hline
\end{tabular}


ligeramente la proporción de niños inscritos en el grupo control y disminuyendo en el grupo intervenido (12\% $\mathrm{p}=0,25 ;-4 \% \mathrm{p}=1,00$, respectivamente). Esto llamó especialmente la atención, ya que el objetivo de aumentar la inscripción a los talleres que se estaban impartiendo en las escuelas era una meta muy concreta, medible y, a nuestro juicio, realista.

Lo observado en este estudio es concordante con las conclusión de una reciente revisión sistemática publicada en la Cochrane Database of Systematic Reviews (21), donde se vió que algunas intervenciones a nivel escolar son efectivas para aumentar el tiempo de duración de la actividad física en niños, pero que no existe evidencia convincente de que estas intervenciones sean efectivas en aumentar el porcentaje de niños activos. Otra revisión del año 2007 (6) mostró, que existen intervenciones efectivas, especialmente a nivel escolar, para aumentar el tiempo de actividad física, pero no hace referencia al porcentaje de niños que la realiza.

Por otro lado la reducción de los minutos diarios de televisión durante la semana fue el resultado más favorable de acuerdo a los objetivos que se plantearon al inicio. Existen varias hipótesis que muestran el impacto de la televisión en el estado nutricional de los niños. Una de ellas postula que la televisión aumenta la probabilidad de consumir bebidas azucaradas y snacks salados, y disminuye la de consumir frutas (22). También se ha visto que la televisión disminuye los niveles de

\section{TABLA 2}

Etapas de cambio en el grupos intervenido y control al inicio del estudio

\begin{tabular}{|c|c|c|c|}
\hline & $\begin{array}{l}\text { Control } \\
(\mathrm{N}=\mathbf{2 5})\end{array}$ & $\begin{array}{c}\text { Intervenido } \\
\quad(\mathbf{N}=\mathbf{2 5})\end{array}$ & $\mathbf{p}$ \\
\hline \multicolumn{4}{|c|}{ Etapa de cambio colación n(\%) } \\
\hline Pre contemplación & $3(12,0)$ & $1(4,0)$ & \multirow{5}{*}{0.212} \\
\hline Contemplación & $3(12,0)$ & $1(4,0)$ & \\
\hline Preparación & $8(32,0)$ & $7(28,0)$ & \\
\hline Acción & $6(24,0)$ & $5(20,0)$ & \\
\hline Mantención & $5(20,0)$ & $11(44,0)$ & \\
\hline \multicolumn{4}{|c|}{ Etapa de cambio dinero $\mathrm{n}(\%)$} \\
\hline Pre contemplación & $0(0,0)$ & $2(8,0)$ & \multirow{5}{*}{0.081 \% } \\
\hline Contemplación & $1(4,0)$ & $2(8,0)$ & \\
\hline Preparación & $11(44,0)$ & $15(60,0)$ & \\
\hline Acción & $3(12,0)$ & $1(4,0)$ & \\
\hline Mantención & $10(40,0)$ & $5(20,0)$ & \\
\hline \multicolumn{4}{|c|}{ Etapa de cambio actividad física n(\%) } \\
\hline Pre contemplación & $4(16,0)$ & $3(12,0)$ & \multirow{5}{*}{0.936} \\
\hline Contemplación & $5(20,0)$ & $5(20,0)$ & \\
\hline Preparación & $11(44,0)$ & $11(44,0)$ & \\
\hline Acción & $4(16,0)$ & $4(16,0)$ & \\
\hline Mantención & $1(4,0)$ & $2(8,0)$ & \\
\hline \multicolumn{4}{|c|}{ Etapa de cambio televisión n(\%) } \\
\hline Pre contemplación & $4(16,0)$ & $1(4,0)$ & \multirow{5}{*}{0.298} \\
\hline Contemplación & $4(16,0)$ & $6(24,0)$ & \\
\hline Preparación & $10(40,0)$ & $13(52,0)$ & \\
\hline Acción & $3(12,0)$ & $2(8,0)$ & \\
\hline Mantención & $4(16,0)$ & $3(12,0)$ & \\
\hline
\end{tabular}


actividad física y, por lo tanto, el gasto energético (23). Otra hipótesis es que la televisión se ha asociado con menos horas de sueño, lo que a su vez se ha asociado al desarrollo de obesidad en los niños (24). Además está el factor publicitario que llama y genera incentivos para el consumo de alimentos ricos en azúcar y grasas (25), especialmente en niños obesos (26).

Al revisar el posible impacto de una disminución de 90 minutos en el tiempo destinado a ver televisión, algunos estudios han detectado cambios favorables en el IMC $(-0,45 \mathrm{~kg} / \mathrm{m} 2 ; \mathrm{p}=0,002)$, circunferencia de cintura $(2,3 \mathrm{~cm} ; \mathrm{p}<0,001)$ y pliegue tricipital $(-1,47 \mathrm{~mm}$; $\mathrm{p}=0,002)$ en niños con una disminución de 30 a 55 minutos diarios (27), mientras que otro estudio mostró que con una disminución de 145 minutos diarios de televisión y computador el IMC disminuye en $-0,24 \mathrm{~kg} /$ $\mathrm{m} 2(\mathrm{p}<0,05)$ y la ingesta de energía en $-180 \mathrm{kcal} / \mathrm{d}$ $(\mathrm{p}<0,05)(28)$. Sin embargo, aun hay escasa evidencia que evalúe la prevalencia de obesidad como variable dependiente, por lo que se necesitan más estudios para emitir conclusiones al respecto.
En relación a las variables secundarias (intención de cambio de conducta), resultados similares se observaron en un estudio que evaluó la disposición al cambio en padres de niños entre 5 y 10 años, luego de una intervención de 3 meses para aumentar actividad física y consumo de frutas y verduras (29). En este sentido, los resultados de este estudio apoyan la conclusión de que el trabajo con los padres de forma aislada, como suele ocurrir en la práctica clínica pediátrica, tendría resultados modestos y requeriría de otras estrategias y de otros contextos para ser efectivo.

Nuestra experiencia ratifica que el modelo transteórico tiene elementos positivos para el cambio de conducta, sin embargo, su extensión a la población general es un aspecto que debe seguir siendo estudiado, principalmente por las dificultades técnicas que implica su aplicación. Entre las dificultades se pueden mencionar la falta de capacitación del personal de salud y/o educación y los escasos tiempos disponibles en la atención necesarios para su desarrollo. Se han planteado otras estrategias para el cambio de conducta como el Consejo

TABLA 3

Dirección de cambio en las variables de interés del grupo intervenido y control

\begin{tabular}{|c|c|c|c|}
\hline & $\begin{array}{l}\text { Control } \\
(\mathrm{N}=24)\end{array}$ & $\begin{array}{l}\text { Intervenido } \\
\qquad(\mathbf{N}=25)\end{array}$ & $\mathbf{p}$ \\
\hline \multicolumn{4}{|l|}{ Lleva colación n(\%) } \\
\hline Sin cambio & $22(91,7)$ & $22(88,0)$ & $1.00 \ddagger$ \\
\hline Cambio negativo & $1(4,2)$ & $1(4,0)$ & \\
\hline Cambio positivo & $1(4,2)$ & $2(8,0)$ & \\
\hline \multicolumn{4}{|l|}{ Tipo de colación n(\%) } \\
\hline Sin cambio & $9(40,9)$ & $13(61,9)$ & $0.202 \ddagger$ \\
\hline Cambio negativo & $5(22,7)$ & $1(4,8)$ & \\
\hline Cambio positivo & $8(36,4)$ & $7(33,3)$ & \\
\hline \multicolumn{4}{|l|}{ Lleva dinero $\mathrm{n}(\%)$} \\
\hline Sin cambio & $17(70,8)$ & $18(72,0)$ & $1.00 \ddagger$ \\
\hline Cambio negativo & $2(8,3)$ & $1(4,0)$ & \\
\hline Cambio positivo & $5(20,8)$ & $6(24,0)$ & \\
\hline \multicolumn{4}{|c|}{ Inscripción taller polideportivo n(\%) } \\
\hline Sin cambio & $21(87,5)$ & $24(96,0)$ & $0.170 \ddagger$ \\
\hline Cambio negativo & $0(0,0)$ & $1(4,0)$ & \\
\hline Cambio positivo & $3(12,5)$ & $0(0,0)$ & \\
\hline TV. $x(D E)$ & $-37,72(77,72)$ & $-90,0(89,16)$ & $0,032 \dagger$ \\
\hline
\end{tabular}


Breve (30), cuyo potencial radica en su aplicabilidad en tiempos realistas de atención, aunque su eficacia y su efectividad son discutibles.

\section{Limitaciones}

La principal limitación de este estudio es el reducido tamaño muestral y en consecuencia el bajo poder estadístico. Si bien se pueden encontrar en la literatura estudios con tamaños muestrales similares al nuestro (31, 32), existen dificultades con el manejo estadístico de los datos, especialmente cuando se analizan categorías y no datos a escala continua. Otro aspecto que pudiera afectar la interpretación de los resultados es el auto reporte de los datos, lo cual puede estar sujeto a sesgos o errores.

Por último la asignación no aleatoria a los grupos intervenidos y control pudiera haber, influido en los resultados.

\section{CONCLUSIONES}

El uso de consejería mostró una tendencia favorable al cambio en algunas de las conductas evaluadas. Si bien este estudio piloto provee resultados promisorios, se requieren de nuevos estudios de mejor calidad metodológica y en contextos reales de implementación para definir si el trabajo con madres/cuidadoras de niños obesos y el uso del modelo transteórico debieran ser incorporados a nivel poblacional para modificar conductas alimentarias y de actividad física en niños.

\section{RESUMEN}

La obesidad es el principal problema nutricional de la población infantil en Chile. La modificación de conductas a través del modelo de etapas de cambio se plantea como una alternativa de prevención de bajo costo y de mayor impacto respecto al modelo informativo. El propósito del estudio fue evaluar el efecto de la Consejería en Vida Sana en el cambio de conducta de niños obesos entre 6 y 9 años de escuelas municipalizadas de la comuna de Macul, Región Metropolitana. Para esto se realizó un estudio piloto con diseño cuasi-experimental que incluyó a las madres/cuidadoras $(\mathrm{N}=50)$ de estos niños. La intervención consistió en tres sesiones de consejería

\section{TABLA 4}

Variación en las etapas de cambio en los grupos intervenido y control

\begin{tabular}{|c|c|c|c|}
\hline & $\begin{array}{l}\text { Control } \\
(\mathrm{N}=24)\end{array}$ & $\begin{array}{l}\text { Intervenido } \\
\qquad(\mathrm{N}=\mathbf{2 5})\end{array}$ & $\mathbf{p}$ \\
\hline \multicolumn{4}{|l|}{ Colación n(\%) } \\
\hline Sin cambio & $15(62,5)$ & $15(60,0)$ & $0.837 \ddagger$ \\
\hline Cambio negativo & $3(12,5)$ & $2(8,0)$ & \\
\hline Cambio positivo & $6(25,0)$ & $8(32,0)$ & \\
\hline \multicolumn{4}{|l|}{ Dinero $n(\%)$} \\
\hline Sin cambio & $18(75,0)$ & $17(68,0)$ & $0.206 \neq$ \\
\hline Cambio negativoa & $2(8,3)$ & $0(0,0)$ & \\
\hline Cambio positivo & $4(16,7)$ & $8(32,0)$ & \\
\hline \multicolumn{4}{|l|}{ Actividad física n(\%) } \\
\hline Sin cambio & $20(83,3)$ & $15(60,0)$ & $0.149 \ddagger$ \\
\hline Cambio negativo & $1(4,2)$ & $5(20,0)$ & \\
\hline Cambio positivo & $3(12,5)$ & $5(20,0)$ & \\
\hline \multicolumn{4}{|l|}{ Televisión n(\%) } \\
\hline Sin cambio & $18(75,0)$ & $13(52,0)$ & $0.329 \ddagger$ \\
\hline Cambio negativo & $1(4,2)$ & $2(8,0)$ & \\
\hline Cambio positivo & $5(20,8)$ & $10(40,0)$ & \\
\hline
\end{tabular}

†Test exacto de Fisher.

Se considera cambio positivo el pasar de las etapas de pre-contemplación, contemplación o preparación, a las etapas de acción o mantención.

Se considera cambio negativo pasar de acción o mantención, a pre-contemplación, contemplación o preparación. 
en el hogar a madres/cuidadoras durante 16 semanas. Los resultados indican que la consejería contribuyó a que los niños del grupo intervenido disminuyeran el tiempo diario de televisión y mejoraran la calidad nutricional de sus colaciones, comparado a lo observado en el grupo control. Se recomienda que se confirme la efectividad de la consejería como estrategia de prevención en un estudio aleatorio con un tamaño muestral mayor.

Palabras clave: Consejería; obesidad infantil; conducta alimentaria; actividad física; madres/cuidadoras

Dirigir la correspondencia a:

Sr.
Álvaro Castillo-Carniglia
INTA, Universidad de Chile
Av. El Líbano 5524
Casilla 138-11, Santiago
Chile
Teléfono: 56-2-2052653
Fax: 56-2-2214030
e-mail: aecastillo@med.uchile.cl

Agradecimientos: A la Corporación de Educación de la Municipalidad de Macul, Santiago, a las escuelas de Macul y a las madres y familias que participaron en el proyecto.

Financiamiento: Este proyecto fue financiado por la Beca Dr. Abraham Stekel (INTA, Universidad de Chile) y Tresmontes Lucchetti S.A.

\section{BIBLIOGRAFÍA}

1. Kain J, Uauy R, Lera L, Taibo M, Espejo F, Albala C. [Evolution of the nutritional status of six years old Chilean children (1987-2003)]. Rev Med Chil 2005;133:1013-20.

2. Junta Nacional de Auxilio Escolar y Becas de Chile. www.junaeb.cl. Revisado en Abril de 2009.

3. Ministerio de Salud Chile. Objetivos Sanitarios para la Década 2000-2010: resumen ejecutivo. El Vigía 2002;5.

4. Ministerio de Salud. Departamento de Estadística e Información de Salud. Santiago: Gobierno de Chile, 2008.

5. Alexandrov AA, Maslennikova GY, Kulikov SM, Propirnij GA, Perova NV. Primary prevention of cardiovascular disease: 3 -year intervention results in boys of 12 years of age. Prev Med 1992;21:53-62.

6. van Sluijs EM, McMinn AM, Griffin SJ. Effectiveness of interventions to promote physical activity in children and adolescents: systematic review of controlled trials. BMJ 2007;335:703.

7. Lizarraga S, Ayarra M. Entrevista motivacional.
ANALES Sis San Navarra 2001;24: 43-53.

8. Prochaska JO. Why do we behave the way we do? Can J Cardiol 1995;11 Suppl A:20A-25A.

9. Albala C, Olivares S, Sánchez H, Bustos N, Moreno X. Consejería en Vida Sana: Manual de Apoyo. Instituto de Nutrición y Tecnología de los Alimentos. 2004:pp 1-39.

10. Green LW. What can we generalize from research on patient education and clinical health promotion to physician counseling on diet? Eur J Clin Nutr 1999;53 Suppl 2:S9-18.

11. Kain J, Concha F, Salazar G, et al. Prevención de obesidad en preescolares y escolares de escuelas Municipales de una Comuna de Santiago: proyecto piloto 2006. ALAN 2009;59:139-46.

12. The WHO Child Growth Standards, Growth reference 5-19 years. 2007.

13. Olivares S, Kain J, Lera L, Pizarro F, Vio F, Moron C. Nutritional status, food consumption and physical activity among Chilean school children: a descriptive study. Eur J Clin Nutr 2004;58:1278-85.

14. 5 a Day for Better Health Program. In: OfficeMonograph GP, ed. Washington DC: National Institutes of Health/National Cancer Institute, 2004.

15. El Nivel Socio Económico Esomar: Manual de Aplicación. Santiago: Adimark, 2000.

16. Olivares S, Zacarias I, Domper A. Educación en Alimentación y Nutrición para la Enseñanza Básica. http://www.inta.cl/Consumidor/Nuevas/guia_escolar.pdf 2007.

17. Zacarias I, González D. Comer verduras y frutas es más fácil de los que usted piensa. http://www. inta.cl/Consumidor/5aldia/comer_verduras_y_frutas_2006.pdf 2005.

18. Olivares S, Zacarías I, Lera L, Leyton B, Durán R, Vio F. Estado Nutricional y consumo de Alimentos Seleccionados en Escolares de la Región Metropolitana: Línea Base para un Proyecto de Promoción del Consumo de Pescado. Rev Chil Nutr 2005;32.

19. Burrows T, Warren JM, Baur LA, Collins CE. Impact of a child obesity intervention on dietary intake and behaviors. Int J Obes (Lond) 2008;32:1481-8.

20. Jahns L, Siega-Riz AM, Popkin BM. The increasing prevalence of snacking among US children from 1977 to 1996 . J Pediatr 2001;138:493-8.

21. Dobbins M, De Corby K, Robeson P, Husson H, Tirilis D. School-based physical activity programs for promoting physical activity and fitness in children and adolescents aged 6-18. Cochrane Database Syst Rev 2009:CD007651.

22. Utter J, Scragg R, Schaaf D. Associations between 
television viewing and consumption of commonly advertised foods among New Zealand children and young adolescents. Public Health Nutr 2006; 9:606-12.

23. Swinburn B, Shelly A. Effects of TV time and other sedentary pursuits. Int J Obes (Lond) 2008;32 Suppl 7:S132-6.

24. Wells JC, Hallal PC, Reichert FF, Menezes AM, Araujo CL, Victora CG. Sleep patterns and television viewing in relation to obesity and blood pressure: evidence from an adolescent Brazilian birth cohort. Int J Obes (Lond) 2008;32:1042-9.

25. Olivares S, Albala C, Garcia F, Jofre I. Television publicity and food preferences of school age children of the metropolitan region. Rev Med Chil 1999;127:791-9.

26. Halford JC, Boyland EJ, Hughes GM, Stacey L, McKean S, Dovey TM. Beyond-brand effect of television food advertisements on food choice in children: the effects of weight status. Public Health Nutr 2008;11:897-904.

27. Robinson TN. Reducing children's television viewing to prevent obesity: a randomized controlled trial. JAMA 1999;282:1561-7.
28. Epstein LH, Roemmich JN, Robinson JL, et al. A randomized trial of the effects of reducing television viewing and computer use on Body Mass Index in young children. Arch Pediatr Adolesc Med 2008;162:239-45.

29. Kubik MY, Story M, Davey C, Dudovitz B, Zuehlke EU. Providing obesity prevention counseling to children during a primary care clinic visit: results from a pilot study. J Am Diet Assoc 2008; 108:1902-6.

30. Albala C, Olivares S, Sánchez H, Bustos N, Moreno X. Consejo Breve en Vida Sana: Guía de Apoyo. Instituto de Nutrición y Tecnología de los Alimentos (INTA), Universidad de Chile:1-17.

31. Vignolo M, Rossi F, Bardazza G, et al. Five-year follow-up of a cognitive-behavioural lifestyle multidisciplinary programme for childhood obesity outpatient treatment. Eur J Clin Nutr 2008;62:1047-57.

32. Foger M, Bart G, Rathner G, Jager B, Fischer H, Zollner-Neussl D. Physical activity, nutritional counseling and psychological guidance in treatment of obese children. A controlled follow-up study over six months. Monatsschr Kinderheilkd 1993;141:491-7. 